\title{
A Mechanical Simulator of Tongue-Palate Compression to Investigate the Oral Flow of Non-Newtonian Fluids
}

\author{
Mr. Andrew Redfearn and Dr Ben Hanson
}

\begin{abstract}
Understanding oral processing is of great interest for the food \& drink industry, and for many groups of people who have difficulties eating $\&$ drinking it is of critical importance for their health and quality of life. This paper describes a simulator which bridges a gap in experimental capability between idealised laboratory-based materials-characterisation studies and human investigations which lack detailed objective measurement. This novel in-vitro mechanical oral simulator was constructed to simulate the in-mouth compression of a bolus of food or thick drink between the tongue and hard palate. Tongue compliance was matched to physiological values. A stepper motor-controlled platform reproduces motion of the tongue to compress a fluid bolus against a palate, propelling it posteriorly. The palate surface contains 5 pressure transducers. Internal bolus flow is imaged using a laser-lit particle image velocimetry (PIV) system at 1000 frames per second. Each component of the simulator was calibrated. Example results are included from a case-study demonstrating differences in pressure-flow characteristics between two model food materials created with starch and gum. Consistent with in-vivo studies, pressure was higher anteriorly (bolus tail) than posteriorly near ejection into the oropharyngeal space. Bolus transport, with intra-bolus shear rates $0-40 \mathrm{~s}^{-1}$, required relatively low tongue pressure: $0.5-1.4 \mathrm{kPa}$ for both the boluses. However, clearing the bolus $(<0.5 \mathrm{~mm}$ residual coating) required much higher pressure $(5.4 \mathrm{vs} 1.7 \mathrm{kPa})$ for the example starch-based material vs the gum-based sample despite both having equal apparent viscosity of $0.47 \mathrm{~Pa} . \mathrm{s}$ at $50 \mathrm{~s}^{-1}$. The simulator has thus demonstrated the ability to quantify how rheologicallydifferent materials may behave differently within the mouth.
\end{abstract}

Index Terms-Dysphagia, swallow, particle image velocimetry, oral processing, food engineering.

\section{INTRODUCTION \& BACKGROUND}

\section{A. Rationale and Aim}

Eating and drinking are fundamental activities of our daily lives, yet the biomechanical processes are relatively unknown (e.g. in comparison to cardiovascular or skeletal biomechanics). Such knowledge is of commercial importance to the food \& drink industry, arguably the largest of all industrial sectors globally. But the importance of in-mouth behaviour of foods \& drinks is more than financial: a wide range of individuals with physical and/or neurological disabilities find eating \& drinking to be a huge challenge to their health and wellbeing. These disorders can often be managed successfully by texture modification of foods and drinks [1], but although this is a very widespread practice (up to $55 \%$ in residential care homes [2]) the clinical evidence base is very small [3]. Objective research so far has faced significant challenges: in-vivo measurement tools are invasive, restrictive or low-quality in resolution and

Submitted for peer review on $\mathrm{xx} / \mathrm{xx} / 2017$. This work is partly supported by an educational grant from Fresenius-Kabi Ltd, who also supplied materials. precision; in-vitro studies are often too idealised, and in-silico simulations generally have to use simplified models of fluidstructure behaviour which potentially exclude critical features of non-Newtonian drinks or complex foods.

The aim of this research was to create a physical simulator for physiological in-mouth compression of a bolus of food or thickened drink in order to quantify the intrabolus pressure gradients as well as visualising the internal flows simultaneously.

\section{B. Measurement and assessment of Bolus Rheology}

Commercial assessment of food and drink textures regularly includes the use of trained sensory evaluation panels, however these are relatively costly and unavoidably subjective which limits their authority and wider applicability, especially internationally [4], [5]. Rheometers and texture analysers are considered the objective gold standard in laboratory research and have unquestionable abilities to characterise food \& drinks as materials. However, these typically apply test conditions which are designed to be well-controlled from a mechanical perspective (e.g. constant shear rate) rather than attempting to replicate physiological motions or interface conditions. Therefore they do not provide information about how a complete bolus of a material deforms when pressure is applied. A further limitation of most laboratory instruments is that they are generally unable to provide information about the internal flow of materials, instead measuring surface boundary conditions (for example torque \& displacement) and relying on models to assume the sample deformation.

The simulator described here has been created to measure the pressure-flow characteristics - rheology - of whole boluses in a simulated situation of compression between the tongue and palate. This is made possible only by the simultaneous measurement of fluid pressure and fluid flow.

\section{Flow Involved in Oral Processing}

For decades, experimental studies using trained panels have attempted to identify a characteristic shear rate which best represents oral processing and swallowing, resulting in values from $10 \mathrm{~s}^{-1}$ [5] to $50 \mathrm{~s}^{-1}$ [6], [7] to $500 \mathrm{~s}^{-1}$ [8]. The oral flow behaviour of both Newtonian and non-Newtonian solutions was investigated in-vitro by Mowlavi et al. (2016) [9] who revealed that several different states and rates of flow are involved, meaning that the internal shear rate will vary throughout the bolus at any instant.

In-vivo measurement devices [10], videofluoroscopy [11] and ultrasound [9] have provided some indications of pressures, shear rates and bolus speeds involved, but with limited clarity and resolution, and the invasiveness of these methods is likely

Andrew Redfearn and B Hanson are with the Department of Mechanical Engineering, University College London, London, UK (e-mail: a.redfearn.12@ucl.ac.uk; ben.hanson@ucl.ac.uk). 
to affect the swallow process itself. There have been relatively few attempts to model the fluid dynamics computationally and the models imposed are generally quite idealised, e.g. assuming compression between two parallel plates [12]-[14]. The interaction between bolus and oral surfaces in-vivo will depend on saliva quantity and quality as well as the bolus properties; a degree of partial slip (and hence, plug flow) can be very significant as it reduces the need for shear throughout the bolus and increases the overall bolus velocity, as simulated computationally by Nicosia [13]. Plug flow was also observed in a theoretical model which considered the influence of saliva on bolus transportation in the pharynx and oesophagus [15].

\section{Physical Models}

This simulator aims to bridge a gap between idealised models (laboratory materials characterisation, computer simulations) and in-vivo studies; in this context there are only a few comparable test rigs, as follows. Mackley and colleagues [16] constructed a mechanical model aiming to reproduce peristaltic flow in which a bolus is confined to a flexible plastic tube and a hard, cylindrical roller imitates the movement of the tongue along a curved palate surface, propelling the bolus posteriorly. A constant torque is applied and the resulting motion is measured. This has been compared to ultrasound images invivo [9], [17], finding them to be in "geometrical, kinematic and dynamic agreement". A similar "slip extrusion test" [18] draws a bolus through a pair of rigid rollers, measuring the force exerted on the rollers by the bolus. Dirven and colleagues [19][21] developed a soft-robotic (pneumatic) model to simulate the peristaltic motion of the oesophagus; a manometry catheter measures pressure gradients from the bolus head to tail. The process of bolus preparation by mastication has also been simulated (e.g. [22], [23]) however that is outside the scope of this simulator: Table 1.

The simulator described herein aims to map pressure from bolus head to tail, since this is seen as critical to understanding oral sensation and the pressures required to swallow semi-solid materials. Further, of the in-vitro simulators reviewed, none directly measure the internal bolus deformation but that is the aim of this simulator, guided by the recommendation of Nicosia [13], "to employ methods from experimental fluid mechanics, such as particle image velocimetry (PIV), to map the velocity field and shear rate during swallowing."

TABLE 1

SCOPE: PHYSIOLOGICAL SWALLOWING CHARACTERISTICS REPRODUCED AND MEASURED

\begin{tabular}{ll}
\hline \hline Characteristics included & Not included/viable currently \\
\hline Oral cavity dimensions & $\begin{array}{l}\text { Texture of tongue, palate and } \\
\text { buccal surfaces }\end{array}$ \\
\hline Bolus volume and shape & Lubricating saliva layer \\
\hline $\begin{array}{l}\text { Controllable swallow motion; } \\
\text { pressure is coupled to motion }\end{array}$ & $\begin{array}{l}\text { Independently-variable pressure } \\
\text { during a swallow }\end{array}$ \\
\hline $\begin{array}{l}\text { Measurement of intra-bolus flow; } \\
\text { fluid pressure on palatal surface }\end{array}$ & Wall shear stresses \\
\hline & Testing of thin Newtonian fluids
\end{tabular}

\section{Materials AND MethodS}

Figure 1 below shows the overall structure of the mechanical oral simulator which was created; each element is described in further detail in the following sub-sections. a)

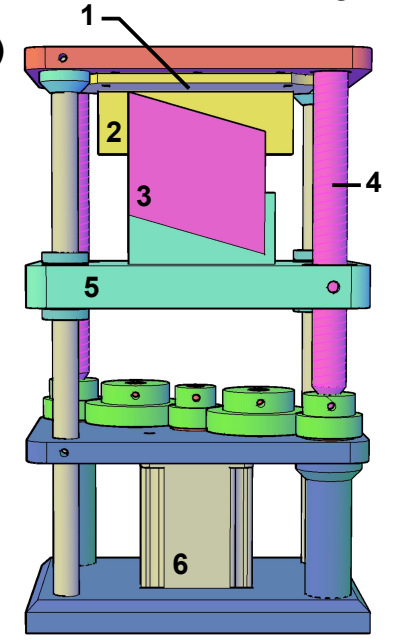

b)

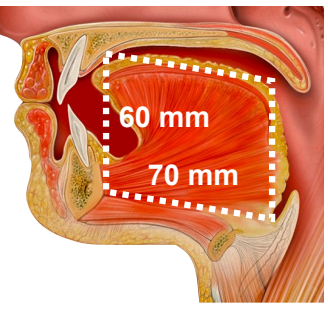

1 Clear acrylic palate

2 Clear acrylic wall

3 Silicone Tongue

4 Lead screw

5 Moving platform

6 Stepper motor c)

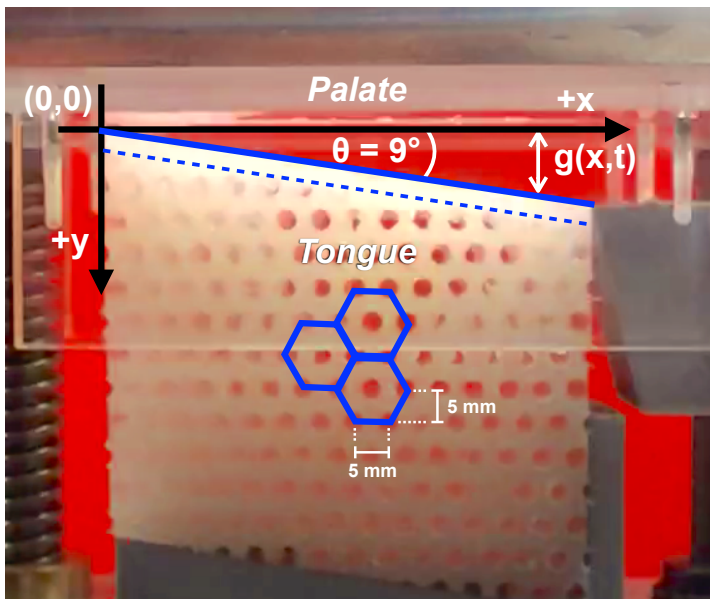

Fig. 1. a) Components of the mechanical oral simulator. The moving platform rises to compress the silicone tongue against the clear acrylic palate. b) Illustrative sagittal view of human oral cavity with model tongue dimensions overlaid. c) Model's oral cavity with dimensions and geometry nomenclature. The variable gap between the tongue and palate surfaces is defined as $g(x, t)$. Porous structure of tongue is described in section (A)

\section{A. Tongue Characteristics}

The tongue dimensions shown in Figure $1 \mathrm{~b}$ are based on previously-published analyses of human anatomy from ultrasound [24], [25] and MR images [26]. Clinical videofluoroscopic swallow studies show the tongue surface declining posteriorly prior to a swallow [27]-[29]; the trapezoidal shape shown in figure 1 was chosen to approximate this slope and maintain equal depth of the tongue along the anterior-posterior $(\mathrm{x})$ dimension.

The lateral dimension $30 \mathrm{~mm}$ was chosen to provide sufficient tongue width for full development of bolus flow and for measurement transducers described below.

The material properties of the physiological tongue are complex and variable, being an active muscle. Ishihara et al., (2013) [30] measured the Young's modulus (E) of tongues of eight human subjects (average age 32.4) using a digital 
micrometer with attached pressure sensor to compress the tongues by $20 \%$ : tongues in a tensed state -i.e. when swallowing- measured $122.5 \pm 58.5 \mathrm{kPa}$ (note the large standard deviation). In order to achieve a model tongue with a specific Young's modulus, silicone (Smooth-On Inc., Macungie, PA, $\mathrm{USA} ; \mathrm{E}=370 \mathrm{kPa}$ ) was moulded into a porous hexagonal grid structure as shown in Figure 1c. With $3 \mathrm{~mm}$-diameter pores in a $5 \mathrm{~mm}$ hexagonal grid, volume fraction of $28 \%$, the resulting structure exhibited a linear stress-strain relationship $(E=132$ $\mathrm{kPa}, \mathrm{r}^{2}=0.998$ ) in compression testing (zwickiLine Z5.0, Zwick Roell, Ulm, Germany). This was assessed up to the maximum strain involved in the simulator, 0.28 , which occurs when the tongue is compressed against the palate. Prior to tongue-palate contact, when the tongue is propelling the bolus, the strain is far smaller. The upper surface is free from pores to a depth of $3 \mathrm{~mm}$ to ensure an even pressure is applied. In future, various textures and/or saliva coatings can be applied instead of this smooth surface.

\section{B. Swallow Pressure}

Physiologically, the pressure to drive a swallow is provided by the tongue and that is also the case in the simulator: the pressure applied is proportional to the compression of the porous silicone in contact with the bolus and palate. Thus pressure and motion are coupled in this simulator by the tongue surface gradient, $\theta$, which results in a pressure waveform moving posteriorly at a constant rate when vertical compression rate is constant. Previously-published in-vivo measurements were used to establish a range of appropriate pressure values (Table 2 ) for healthy and weakened conditions ( $\mathrm{P}_{\max }$ from 2-36 $\mathrm{kPa})$ [10], [31], [35]-[40] which can be re-created by changing the tongue surface angle and Young's modulus. The tongue surface angle, $\theta$, also determines the bolus volume held in the oral cavity (2 - $15 \mathrm{ml})$ [32]-[35]. The example results shown in this paper were obtained with $\theta=9^{\circ}$, in order to achieve $\mathrm{P}_{\max }=$ $12.5 \mathrm{kPa}$ (middle of physiological range) and an oral cavity volume of $11 \mathrm{ml}$.

\section{Physiological motion}

During a normal swallow, the tongue elevates and contacts the hard palate initially at the front then sweeps posteriorly to propel the bolus. In-vivo, this sweeping motion has been measured with pressure sensors at three locations along the palate [36], [37], by computerised tomography (CT) [38], MRI [39], and by contact sensors on the palate (electropalatography) which have described the sweeping pattern occurring at a relatively constant speed [10], [40]-[42]. Data from these studies (involving a variety of boluses) were analysed to derive a range of physiological tongue-palate sweep speeds: Table 2. Using stepper motor control (Arduino Uno, Arduino, Torino, Italy), this simulator controls motion at the base of the tongue which results in an anterior-posterior sweeping pattern between tongue and palate. For the studies in this paper, a constant speed was applied to the base of the tongue which resulted in sweepspeeds consistent with in-vivo data for thickened drink boluses [38].

In-vivo, the motion of the tongue is controlled by a complex
TABLE 2

SUMMARY OF SWALLOWING PARAMETERS RELATING TO PHYSIOLOGY, AND EXAMPLE DATA MEASURED FROM THE SIMULATOR IN CASE-STUDY

\begin{tabular}{llll}
\hline \hline Parameter & $\begin{array}{l}\text { Values from } \\
\text { literature }\end{array}$ & References & $\begin{array}{l}\text { Measured from } \\
\text { simulator }\end{array}$ \\
\hline $\begin{array}{l}\text { Sagittal Tongue } \\
\text { Dimensions }\end{array}$ & $\begin{array}{l}70 \times 60 \mathrm{~mm}(\mathrm{w} \\
\mathrm{x} \mathrm{h})\end{array}$ & {$[26],[43]$} & $\begin{array}{l}70 \times 60 \mathrm{~mm} \\
(\mathrm{w} \mathrm{x} \mathrm{h})\end{array}$ \\
\hline $\begin{array}{l}\text { Tongue Elastic } \\
\text { Modulus }\end{array}$ & $125 \pm 55 \mathrm{kPa}$ & {$[30],[43]$} & $132 \mathrm{kPa}$ \\
\hline $\begin{array}{l}\text { Anterior - Posterior } \\
\text { contact point speed }\end{array}$ & $35-328 \mathrm{~mm} \mathrm{~s}^{-1}$ & {$[10],[36]-$} & $\begin{array}{l}116,128 \mathrm{~mm} \mathrm{~s}^{-1} \\
(\mathrm{Gum}, \mathrm{Starch})\end{array}$ \\
\hline $\begin{array}{l}\text { Tongue-Palate } \\
\text { Contact Pressure }\end{array}$ & $2-36 \mathrm{kPa}$ & {$[10],[31]$,} & $12.5 \mathrm{kPa}$ \\
\hline Oral Stage Duration & $320-3036 \mathrm{~ms}$ & {$[40]-[42]$,} & $1000 \mathrm{~ms}$ \\
& & {$[47]$} & \\
\hline Bolus Volume & $2-15 \mathrm{ml}$ & {$[32],[35]$} & $11 \mathrm{ml}$
\end{tabular}

and largely un-mapped physiological feedback loop dependent upon tactile, taste and thermal sensory information. That feedback pathway is absent in this simulator, but the replica tongue deforms passively dependent upon the mechanical resistance of the experimental bolus. The simulator can reproduce trajectories involving variation of speed if required, e.g. for proof-of-concept a trajectory was constructed by digitising published ultrasound data [17] at six way-points (not shown).

\section{Particle Image Velocimetry (PIV) system}

This experimental technique is used to quantify flow in a

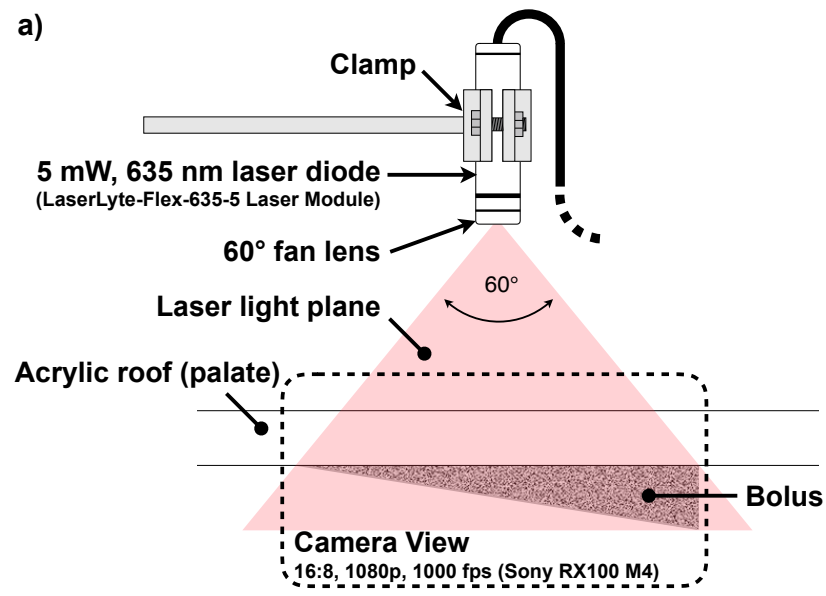

b)

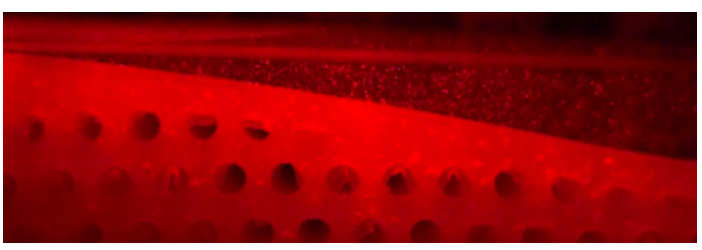

c)

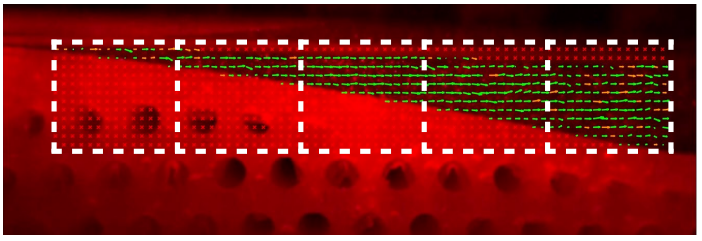

Fig. 2. a) Schematic of PIV setup in sagittal plane. b) A frame of the highspeed footage showing bolus with micro bubbles illuminated by laser. c) Array of velocity vectors calculated using PIV analysis, also shown are the 5 sections of the bolus to be analysed. 
planar cross section of fluid. A plane-focused coherent laser illuminates the fluid and a high-speed camera captures images of this illuminated plane. Typically the clear fluid is doped with reflective seeding particles which are chosen to be small enough (and with matched density) to avoid disturbing the flow, however for the thickened liquids used in much of this work there was sufficient internal texture due to the inherent presence of trapped micro-bubbles of air, and no further seeding was required. Image-processing software (PIVlab [45]) analyses the movement of micro-bubble patterns between pairs of successive images to generate $2 \mathrm{D}$ velocity vector maps of the flow, e.g. Figure 2c; these can be used to calculate shear rates. Further post-processing is carried out using scripts written in Matlab (MathWorks, Natick, MA, USA): first the x-component of velocity is extracted as an average of frame-pairs (e.g. 25 pairs from 172 to $198 \mathrm{~ms}$ in the case-study presented herein). Then, five velocity profiles along $\mathrm{x}$ are calculated for each of the dashed sections shown in Figure 2c, by averaging velocity data in the $y$ direction. Examples of these profiles are presented later in Figure 6.

\section{E. Pressure Sensing}

In-vivo studies have used a pressure pad system attached to the palate [10] to quantify the contact pressure between tongue and palate which provides information about muscular strength and coordination. Unfortunately, this technique is not useful for studying interaction with the bolus nor bolus rheology since the position of the bolus is not known; pressure recordings are not directly attributable to bolus rheology because once tonguepalate contact has been made the bolus is effectively absent. However, since the in-vitro simulator herein is able to visualise the position of the bolus, it is able to study pressure and flow within the bolus.

Figure 3 shows one of the five palate-mounted pressure sensors spaced at $15-\mathrm{mm}$ intervals anteriorly-posteriorly. The presence of a protective latex membrane means the pressure sensed is less than the pressure applied, so each sensor configuration was calibrated experimentally by applying hydrostatic pressure. The relationship between applied pressure and sensor voltage was modelled by a fifth-order polynomial $\left(\mathrm{r}^{2}\right.$

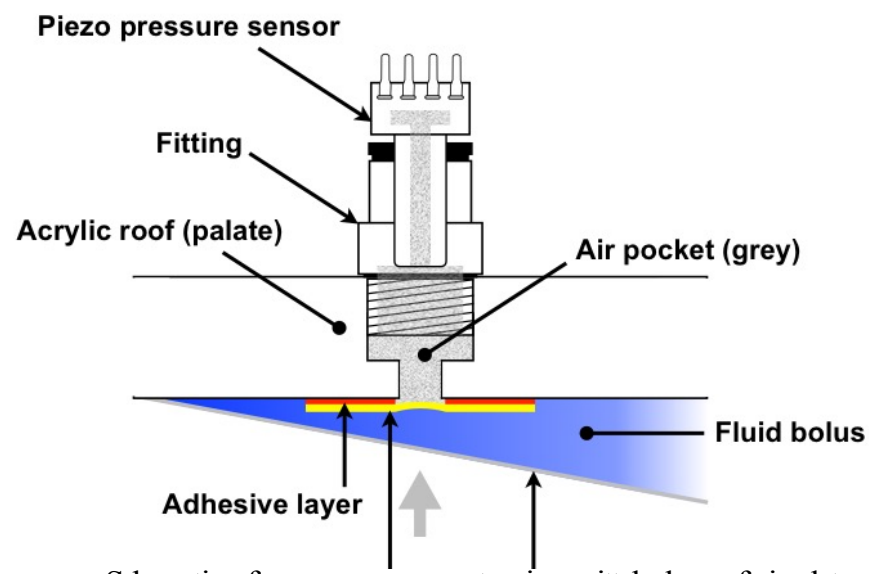

Fig. 3. Schematic of pressure sensor setup in sagittal plane of simulator. Thickness of adhesive layer and latex membrane combined is $0.1 \mathrm{~mm}$
$=0.999)$. A sensed range of $0-1 \mathrm{kPa}$ corresponds to external fluid pressure of $0-16.5 \mathrm{kPa}$, suitable for semi-solid foods and thick liquids; sensors with larger or smaller ranges are able to be swapped depending on the application.

\section{F. Control System}

The microcontroller provided robust, adaptable real-time control of the tongue's vertical motion. Common experimental protocols were programmed, providing an efficient user interface using buttons, a rotary knob and a display screen. Pressure measurements are recorded with a 16-bit data acquisition device (National Instruments USB-6211, NI, Austin, TX, USA) which provides higher resolution and sample rate than is available with the microcontroller. LabVIEW software (National Instruments, Austin, TX, USA) was used for high-level control including selection of swallow duration and synchronisation of each component and measurement (highspeed camera and pressure data).

The experimental procedure begins by setting the initial tongue position shown in figure 1c. A fluid bolus $(11 \mathrm{ml}$ in this example) is loaded into the oral cavity using a syringe and flexible tube then the swallow is immediately triggered. The oral cavity is cleaned manually between each test; the semiautomation allows repeat experiments to be conducted every 5 minutes.

\section{G. Geometrical Image Analysis}

In addition to the fluid PIV analysis, image-processing scripts were created using Matlab (MathWorks, Natick, MA, USA) to capture the position and geometry of the artificial tongue surface through the simulated swallow. The tongue surface is identified through thresholding the camera image and fitting a fifth-order polynomial curve to the boundary between tongue and bolus. The flat palate surface is identified manually on the image (and does not move). Gap width, $\mathrm{g}(\mathrm{x}, \mathrm{t})$, is then defined as the vertical distance between the tongue and palate surface, varying with $\mathrm{x}$-position and time.

\section{RESUltS AND DiscUSSION}

The following example results show a small case-study investigation intending to provide an example data set to complement the descriptions of the individual simulator components. Future publications will focus on the use of the simulator to investigate materials in detail. This case-study compares two different thick liquids which are commonly used by patients with dysphagia (swallowing disorders). These were created by mixing mineral water (Evian $\AA$, Danone) with $(A)$ a thickener based on xanthan gum (Thick \& Easy ${ }^{\mathrm{TM}}$ Clear, Hormel Health Labs) and (B) a thickener based on modified maize starch (Thick \& Easy ${ }^{\mathrm{TM}}$, Hormel Health Labs). Swallow simulations for both liquids were carried out in triplicate.

The shear rheology of each material was studied over a wide range of shear rates $\left(0.1-1000 \mathrm{~s}^{-1}\right)$ using a cone-plate rheometer (TA instruments DHR3, geometry: 60mm-dia and $1^{\circ}$ angle). The two materials have non-Newtonian characteristics: gum being highly shear-thinning (power index of approximately 0.15 ) and starch being less-so (power index: 0.42). The 
concentrations of each thickener were calculated to provide fluids with equal apparent viscosity ( $0.47 \mathrm{~Pa} . \mathrm{s})$ at the shear rate of $50 \mathrm{~s}^{-1}$, in order to investigate the appropriateness of this rate in describing the oral compressive stage of swallowing [46].

Figure 4 shows the bolus pressure recorded at each sensor against time for each test bolus. Pressure data is not shown after the gap width decreases below the minimum measurable width of $0.5 \mathrm{~mm}$ ( $2 \mathrm{x}$ the practical measurement resolution). Below this gap the tongue is considered to be in contact with the palate, accepting that there may be a very thin film of trapped fluid between tongue and palate. Both materials exhibit a pressure gradient along the bolus at any point in time as it is propelled from anterior (higher pressure) posteriorly (lower pressure); this feature is also observed in-vivo [10]. Since the sensor
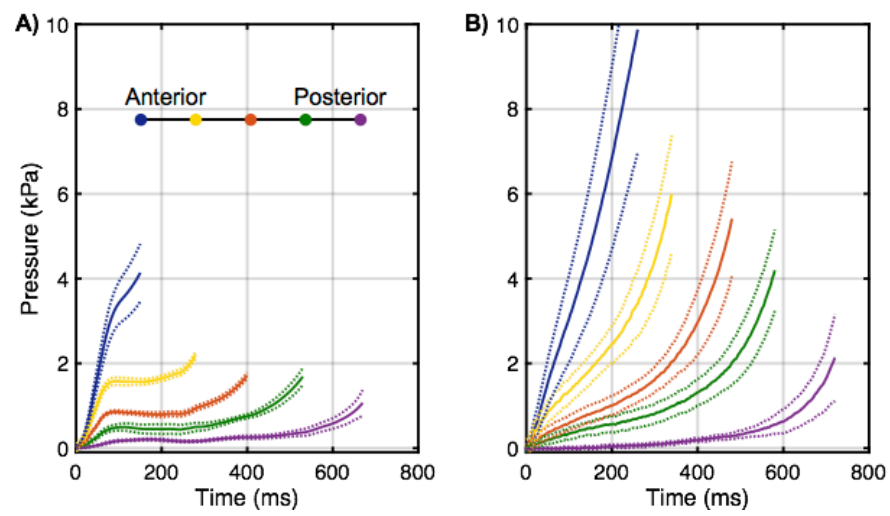

Fig. 4. Pressure data at 5 points on the palate surface for A) Gum bolus and B) Starch bolus with equal apparent viscosity of $0.47 \mathrm{~Pa} . \mathrm{s}$ at $50 \mathrm{~s}^{-1}$. Standard deviation for pressure is shown as dashed line $(n=3)$.

locations are fixed the tongue-palate contact point speed can be derived from Figure 4 as 116 and $128 \mathrm{~mm} \cdot \mathrm{s}^{-1}$ for gum and starch respectively; these values are within the range of published invivo values (Table 2) [17], [40]

Figure 5 shows a comparison of the pressure recorded at the central sensor which reveals differences between the materials in two characteristic features. During the first phase of transport, the pressure retains a relatively constant gradient: for starch (B), the pressure monotonically increases as the gap decreases, whereas gum (A) produces a small initial peak in pressure, which slightly decreases. As the gap closes, the behaviour then tended to deviate, showing a more-rapidly rising pressure; for both materials this occurred when the gap width < $2 \mathrm{~mm}$, Figure 5. In this model, the clearance pressure is defined as the maximum pressure recorded as the bolus is squeezed to a thin film at the minimum measurable gap width of $0.5 \mathrm{~mm}$; For the measurement in Figure 5 this is much higher for the starch bolus (B) at $5.4 \mathrm{kPa}$ compared to $1.7 \mathrm{kPa}$ for gum (A). This difference in the transport \& clearance kinetics of each material is consistent with clinical findings in dysphagia populations of higher levels of post-swallow residue for starchthickened boluses [1]. All pressure values shown in figure 5 are significantly lower than 'maximum swallow pressure' values reported in many in vivo studies [35], [47]. This is because peak values recorded by palate-mounted sensor systems occur after the bolus has been cleared; those peak pressures do not provide data on the pressure required to transport and clear a bolus, thus

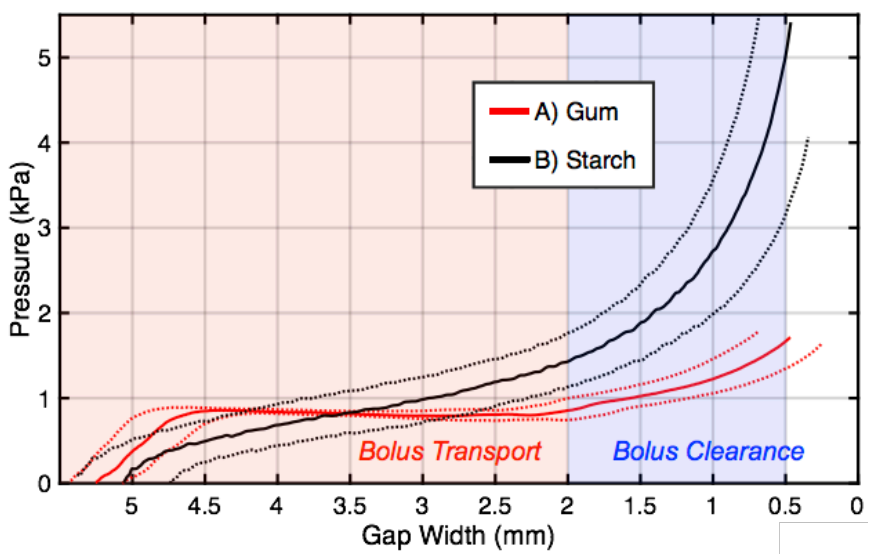

Fig. 5. Pressure Vs gap width for the median pressure sensor. Note: the gap width axis is reversed so that the plot progresses conventionally from left to right as time progresses. Standard deviation for gap width $(0.25 \mathrm{~mm})$ and pressure (variable) is shown as dashed line $(n=3)$.

they are limited in their ability to distinguish between material characteristics in comparison to the in-vitro data available from this simulator. Since dysphagia is often associated with a diminished tongue strength, this simulator provides the opportunity to examine food \& drink materials with the aim of predicting their suitability for a certain patient type.

Figure 6 shows the velocity profiles across the bolus from PIV analysis of 25 frame pairs $(172-198 \mathrm{~ms})$ from each video (at 1,000 f.p.s.). Flow was assumed to be quasi-steady-state during this time period. All velocity profiles indicate partial slip at the palate and tongue surfaces with apparently greater slip at the palate surface, in as far as it has been possible to measure with the $0.25 \mathrm{~mm}$ imaging resolution. In-vivo, the degree of slip is likely to be strongly dependent on the surface texture and the nature of saliva coating, which is not included in this simulator but which is an intended future enhancement. Velocity measurements for the gum bolus (A) are higher overall compared to the starch bolus (B); since the same pressure waveform is applied by the simulator, this result reflects the fact that bolus B is resisting flow more (higher pressure magnitudes recorded) and consequently deforming the compliant tongue more. In comparison, bolus A is transported posteriorly with less pressure and demonstrates more of a plug-flow type profile - this result is consistent with gum-thickened fluids being perceived orally as being "slippery" [48]. Direct comparison with in-vivo oral data is not available, however in-vivo data from the end of the oral phase [9] and the pharyngeal phase [1], [49] have been published; those velocities are higher than these
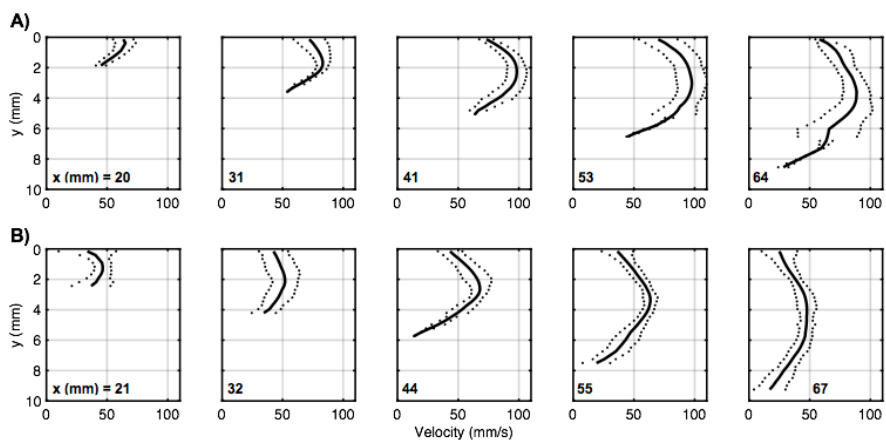

Fig. 6. Velocity profiles across y for five $\mathrm{x}$ locations for A) Gum and B) Starch boluses measured as the average of 24 frame pairs $172-198 \mathrm{~ms}$ from the start of motion. Standard deviation for velocity is shown as dashed line $(n=3)$. 
seen orally, as would be expected (e.g. 100 during tonguepalate compression in-vitro vs $425 \mathrm{~mm} / \mathrm{s}$ at ejection into pharynx in-vivo).

Figure 7 shows the shear rate profile measured at the central $\mathrm{x}$-position. The large variation in shear rates across the bolus confirms the assumption that the shear rate must vary through a bolus during the oral phase of swallowing [13]; zero shear was measured in the centre of the bolus for both material types and the maximum shear rates were up to $40 \mathrm{~s}^{-1}$. While the absolute maximum shear rate will depend on the speed of compression as well as the bolus rheology, it is clear that rheometry measurements at any one shear rate would not represent the full range of fluid flow in practice, in this context, a rate of $50 \mathrm{~s}^{-1}$ (derived from subjective panel studies [7]) was, until recently,

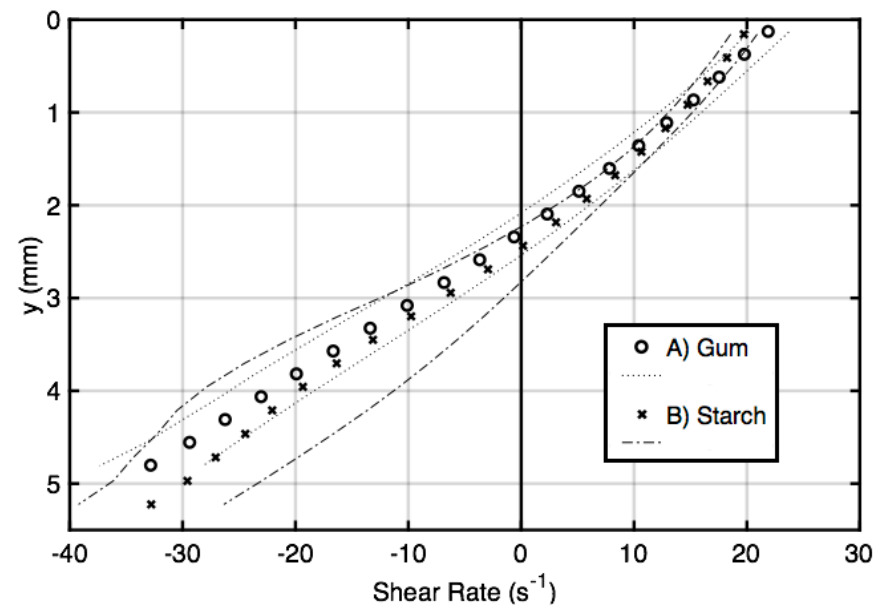

Fig. 7. Internal shear rates for boluses calculated from differentiating thirdorder polynomial models of velocity profiles $\left(\mathrm{r}^{2}=0.996\right.$, A and 0.994 , B). At the central x-position during the period $172-198 \mathrm{~ms}$ from start of motion.

recommended to evaluate thickened drinks [50]. While rheometers can evaluate a fluid over a wide range of shear rates, it has not been possible until now to identify which rates will be relevant in the oral phases of swallowing. The ability of this simulator to measure the range of shear rates throughout the bolus may be useful in predicting the mouthfeel of nonNewtonian fluids such as these used in dysphagia management.

The simulator demonstrated that low bolus pressures (far lower than the maximum tongue-palate contact pressures recorded in an in-vivo swallow) are sufficient to propel thick liquids orally. However, achieving tongue-palate contact and fully clearing a bolus from the mouth requires a significantly increased pressure, strongly dependent on bolus rheology.

\section{CONCLUSIONS}

A mechanical oral simulator has been constructed with the ability to create motions and pressures which are relevant to the oral phase of swallowing. Physiological movement of the tongue (obtained from previously-published ultrasound data) was reproduced using a servo-controlled motor and a simulated tongue was constructed with a bulk compliance which was matched to published physiological values. These elements are controllable providing versatility to simulate a range of healthy and abnormal conditions.
The simulator extends the capabilities of traditional in-vitro materials test apparatus - e.g. rheometers - by providing visualisation of flow through a bolus with a volume and geometry relevant to oral processing. This measurement system provides an advantage over current in-vivo measurement techniques for studying the swallowing process, and may therefore offer a useful quantified objective complement to traditional subjective panel assessments of food and drink.

In an example case-study the measurement systems (pressure transducers and PIV) have demonstrated sufficient resolution to be able to distinguish differences in flow patterns and pressure gradients between two example test fluids having identical apparent viscosity at $50 \mathrm{~s}^{-1}$ but different shear-thinning characteristics. These results predominantly serve to demonstrate the ensemble operation of the simulator but also show that the oral flow of thickened boluses cannot be predicted purely by their viscosity at $50 \mathrm{~s}^{-1}$. Future research will investigate a range of non-Newtonian fluids more comprehensively at different concentrations. Rheological classification of these fluids must include a range of shear rates, specifically those below $50 \mathrm{~s}^{-1}$ in order to understand their behaviour in the oral cavity during swallowing.

This paper demonstrated the capabilities and potential applications for in-vitro swallowing research. Key limitations of the model are the lack of saliva lubrication and unphysiological smooth tongue and palate surfaces. Future research using this simulator will investigate the effect of tongue surface texture (as characterised elsewhere [50]) and saliva coating on the oral surfaces as well as the effect of the speed of tongue motion and tongue compliance.

\section{REFERENCES}

[1] N. Vilardell, L. Rofes, V. Arreola, R. Speyer, and P. Clavé, "A Comparative Study Between Modified Starch and Xanthan Gum Thickeners in Post-Stroke Oropharyngeal Dysphagia," Dysphagia, vol. 31, no. 2, pp. 169-179, 2016.

[2] J. Kayser-Jones and K. Pengilly, "Dysphagia among nursing home residents.," Geriatr. Nurs., vol. 20, no. 2, pp. 77-82, 1999.

[3] J. a Y. Cichero et al., "The Need for International Terminology and Definitions for Texture-Modified Foods and Thickened Liquids Used in Dysphagia Management: Foundations of a Global Initiative," Curr. Phys. Med. Rehabil. reports, vol. 1, pp. 280291, Jan. 2013.

[4] F. Shama, C. Parkinson, and P. Sherman, "Identification Of Stimuli Controlling The Sensory Evaluation Of Viscosity," J. Texture Stud., vol. 4, pp. 102-110, 1973.

[5] A. N. Cutler, E. R. Morris, and L. J. Taylor, "Oral Perception Of Viscosity In Fluid Foods And Model Systems," J. Texture Stud., vol. 14, pp. 377-395, 1983.

[6] F. W. Wood, Psychophysical Studies on the Consistency of Liquid Foods, Conference., no. 27. London: London, Society of Chemical Industry, 1968., 
1968.

[7] M. Akhtar, J. Stenzel, B. S. Murray, and E. Dickinson, "Factors affecting the perception of creaminess of oilin-water emulsions," Food Hydrocoll., vol. 19, no. 3, pp. 521-526, 2005.

[8] A. L. Koliandris, C. Morris, L. Hewson, J. Hort, A. J. Taylor, and B. Wolf, "Correlation between saltiness perception and shear flow behaviour for viscous solutions," Food Hydrocoll., vol. 24, no. 8, pp. 792799, 2010.

[9] S. Mowlavi et al., "In vivo observations and in vitro experiments on the oral phase of swallowing of Newtonian and shear-thinning liquids," J. Biomech., 2016

[10] K. Hori et al., "Newly developed sensor sheet for measuring tongue pressure during swallowing.," $J$. Prosthodont. Res., vol. 53, no. 1, pp. 28-32, Jan. 2009.

[11] C. C. Walczak, C. A. Jones, and T. M. McCulloch, "Pharyngeal Pressure and Timing During Bolus Transit," Dysphagia, 2016.

[12] M. A. Nicosia, "A planar finite element model of bolus containment in the oral cavity," Comput. Biol. Med., vol. 37, no. 10, pp. 1472-1478, 2007.

[13] M. a. Nicosia, "Theoretical Estimation of Shear Rate during the Oral Phase of Swallowing: Effect of Partial Slip," J. Texture Stud., vol. 44, no. 2, pp. 132-139, Apr. 2013.

[14] Y. Meng, M. a. Rao, and a. K. Datta, "Computer Simulation of the Pharyngeal Bolus Transport of Newtonian and Non-Newtonian Fluids," Food Bioprod. Process., vol. 83, no. 4, pp. 297-305, Dec. 2005.

[15] C. De Loubens, A. Magnin, M. Doyennette, I. C. Tréléa, and I. Souchon, "A biomechanical model of swallowing for understanding the influence of saliva and food bolus viscosity on flavor release," $J$. Theor. Biol., vol. 280, no. 1, pp. 180-188, 2011.

[16] M. R. Mackley, C. Tock, R. Anthony, S. a. Butler, G. Chapman, and D. C. Vadillo, "The rheology and processing behavior of starch and gum-based dysphagia thickeners," J. Rheol. (N. Y. N. Y)., vol. 57, no. 6, pp. 1533-1553, 2013.

[17] P. Hayoun, J. Engmann, S. Mowlavi, B. Le Reverend A. Burbidge, and M. Ramaioli, "A model experiment to understand the oral phase of swallowing of Newtonian liquids," J. Biomech., vol. 48, no. 14, pp. 3922-3928, 2015.

[18] G. C. F. Ng, E. M. Gray-Stuart, M. P. Morgenstern, J. R. Jones, N. P. Grigg, and J. E. Bronlund, "The Slip Extrusion Test: A Novel Method to Characterise Bolus Properties," J. Texture Stud., no. December 2016, pp. 1-8, 2017.

[19] S. Dirven, J. Allen, W. (Peter) Xu, and L. K. Cheng, "Soft-robotic esophageal swallowing as a clinicallyinspired bolus rheometry technique," Meas. Sci. Technol., vol. 28, no. 3, p. 35701, 2017.

[20] F. Chen, S. Dirven, W. Xu, S. Member, and X. Li, "Large-Deformation Model of a Soft-Bodied Esophageal Actuator Driven by Air Pressure," vol. 22, no. 1, pp. 81-90, 2017.
[21] M. Z. Zhu, W. L. Xu, S. Member, and L. K. Cheng, "Esophageal Peristaltic Control of a Soft-bodied Swallowing Robot by Central Pattern Generator," IEEE/ASME Trans. Mechatronics, vol. 22, no. 1, pp. 91-98, 2017.

[22] M. Steiner, M. E. Mitsias, K. Ludwig, and M. Kern, "In vitro evaluation of a mechanical testing chewing simulator," Dent. Mater., vol. 25, no. 4, pp. 494-499, 2009.

[23] D. Raabe, K. Alemzadeh, A. J. L. Harrison, and A. J. Ireland, "The chewing robot: A new biologicallyinspired way to evaluate dental restorative materials," Proc. 31st Annu. Int. Conf. IEEE Eng. Med. Biol. Soc. Eng. Futur. Biomed. EMBC 2009, pp. 6050-6053, 2009.

[24] T. H. Shawker, B. Sonies, M. Stone, and B. J. Baum, "Real-time ultrasound visualization of tongue movement during swallowing," J. Clin. Ultrasound, vol. 11, pp. 485-490, 1983.

[25] M. Nakamori et al., "Tongue thickness evaluation using ultrasonography can predict swallowing function in amyotrophic lateral sclerosis patients," Clin. Neurophysiol., 2015.

[26] S. M. Mijailovich, B. Stojanovic, M. Kojic, A. Liang, V. J. Wedeen, and R. J. Gilbert, "Derivation of a finite-element model of lingual deformation during swallowing from the mechanics of mesoscale myofiber tracts obtained by MRI.," J. Appl. Physiol., vol. 109, pp. 1500-1514, Nov. 2010.

[27] M. Stone, "A three-dimensional model of tongue movement based on ultrasound and x-ray microbeam data," J Acoust Soc Am., vol. 87, no. 5, pp. 22072217, 1990.

[28] S. M. Tasko, R. D. Kent, and J. R. Westbury, "Variability in tongue movement kinematics during normal liquid swallowing," Dysphagia, vol. 17, no. 2, pp. 126-138, Jan. 2002.

[29] E. M. Wilson and J. R. Green, "Coordinative organization of lingual propulsion during the normal adult swallow," Dysphagia, vol. 21, no. 4, pp. 226236, Oct. 2006.

[30] S. Ishihara et al., "Compression Test of Food Gels on Artificial Tongue and Its Comparison with Human Test," J. Texture Stud., vol. 44, no. 2, pp. 104-114, Apr. 2013.

[31] C. M. Steele, G. L. Bailey, and S. M. Molfenter, "Tongue pressure modulation during swallowing: water versus nectar-thick liquids," J. speech, Lang. Hear. Res., vol. 53, no. 2, pp. 273-283, 2010.

[32] C. M. Steele and P. Van Lieshout, "Does Barium Influence Tongue Behaviors During Swallowing?," Am. J. Speech-Language Pathol., vol. 14, pp. 27-39, 2005.

[33] W.-Y. Shieh, C.-M. Wang, and C.-S. Chang, "Development of a Portable Non-Invasive Swallowing and Respiration Assessment Device," Sensors, vol. 15, no. 6, pp. 12428-12453, 2015.

[34] E. Kamarunas, G. H. McCullough, M. Mennemeier, and T. Munn, "Oral perception of liquid volume changes with age," J. Oral Rehabil., vol. 42, no. 9, pp. 
657-662, 2015.

[35] W. A. Alsanei and J. Chen, "Studies of the Oral Capabilities in Relation to Bolus Manipulations and the Ease of Initiating Bolus Flow," J. Texture Stud., vol. 45, no. 1, pp. 1-12, Feb. 2014.

[36] Q. Li et al., "Coordination in oro-pharyngeal biomechanics during human swallowing," Physiol. Behav., vol. 147, pp. 300-305, 2015.

[37] J. Yano et al., "Sequential Coordination between Lingual and Pharyngeal Pressures Produced during Dry Swallowing," Biomed Res. Int., 2014.

[38] S. Shibata et al., "Swallowing maneuver analysis using 320-row area detector computed tomography (320-ADCT)," Japanese J. Compr. Rehabil. Sci., vol. 2, pp. 54-62, 2011.

[39] S. Karaçay, E. Akin, M. O. Sayin, and N. Bulakbaşi, "Real time balanced turbo field echo Cine-MRI in the analysis of deglutition events and transit times," $J$. Oral Rehabil., vol. 33, no. 9, pp. 646-653, Sep. 2006.

[40] F. Jack and F. Gibbon, "Electropalatography in the study of tongue movement during eating and swallowing (a novel procedure for measuring texturerelated behaviour)," Int. J. Food Sci. Technol., vol. 30, no. 4, pp. 415-423, 1995.

[41] J. Yano, I. Kumakura, K. Hori, K. I. Tamine, and T. Ono, "Differences in biomechanical features of tongue pressure production between articulation and swallow," J. Oral Rehabil., vol. 39, pp. 118-125, 2012.

[42] C. Peng and R. Miethke, "Ultrasonographic Measurement of Tongue Movement During Swallowing," J. Ultrasound Med., vol. 19, pp. 15-20, 2000.

[43] Y. Payan and P. Perrier, "Synthesis of V-V sequences with a 2D biomechanical tongue model controlled by the Equilibrium Point Hypothesis," Speech Commun., vol. 22, pp. 185-205, 1997.

[44] C. R. Watts and B. Kelly, "The Effect of Bolus Consistency and Sex on Electrophysiological Measures of Hyolaryngeal Muscle Activity During Swallowing," Dysphagia, vol. 30, no. 5, pp. 551-557, 2015.

[45] W. Thielicke and E. J. Stamhuis, "PIVlab - Towards User-friendly, Affordable and Accurate Digital Particle Image Velocimetry in MATLAB," J. Open Res. Softw., vol. 2, 2014.

[46] American Dietetic Association, National Dysphagia Diet: Standardization for Optimal Care. Chicago, IL, 2002.

[47] J. Robbins, R. Levine, J. Wood, E. Roecker, and E. Luschei, "Age Effects on Lingual Pressure Generation as a Risk Factor for Dysphagia," J. Gerontol. Med.
Sci., vol. 50A, no. 5, pp. 257-262, 1995.

[48] Z. Matta, E. Chambers IV, J. M. Garcia, and J. M. G. Helverson, "Sensory Characteristics of Beverages

Prepared with Commercial Thickeners Used for Dysphagia Diets," J. Am. Diet. Assoc., vol. 106, no. 7, pp. 1049-1054, 2006.

[49] L. Rofes et al., "Pathophysiology of oropharyngeal dysphagia in the frail elderly," Neurogastroenterol. Motil., vol. 22, no. 8, pp. 851-859, 2010.

[50] H. Ranc, C. Servais, P.-F. Chauvy, S. Debaud, and S. Mischler, "Effect of surface structure on frictional behaviour of a tongue/palate tribological system," Tribol. Int., vol. 39, no. 12, pp. 1518-1526, Dec. 2006.

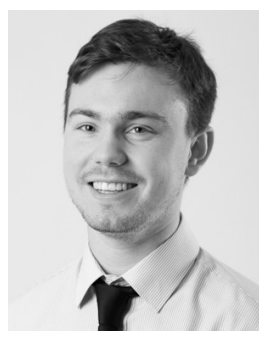

Andrew Redfearn received a $\mathrm{BSc}$ in Physics from the University of Leeds in 2010 and a MSc degree in Mechanical Engineering from University College London in 2013. He is currently working toward a PhD at UCL which investigates the fluid dynamics of thickened drinks used to aid patients with swallowing impairment (Dysphagia).

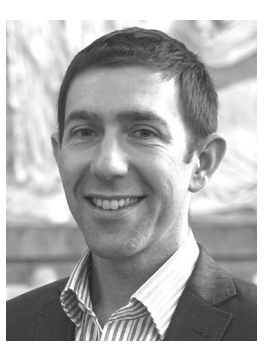

Ben Hanson (PhD, FIMechE, FHEA) is a Senior Lecturer at UCL where he researches biomedical applications of engineering. He has designed miniature devices to measure rheology of fluids in clinical environments, in-situ. $\mathrm{He}$ investigates the rheology of texturemodified foods and drinks which are used in hospitals and home-care environments. Dr Hanson is a board member of the International Dysphagia Diet Standardisation Initiative (IDDSI.org), providing the engineering basis to its international guidelines. 